TITLE:

\title{
Moduli of parabolic stable sheaves
}

$\operatorname{AUTHOR}(S):$

Maruyama, M.; Yokogawa, K.

\section{CITATION:}

Maruyama, M. ...[et al]. Moduli of parabolic stable sheaves. 代数幾何学 シンポジューム記録 1990, 1990: 86-109

ISSUE DATE:

1990

URL:

http://hdl.handle.net/2433/212714

RIGHT: 


\title{
Moduli of Parabolic Stable Sheaves
}

\author{
M.Maruyama K. Yokogawa \\ Department of Mathematics \\ Faculty of Science \\ Kyoto University \\ Kyoto, 606, Japan
}

\section{Introduction}

Since a beautiful relationship between irreclucible unitary representations of the fundamental group $\pi_{1}(X)$ of a compact Riemann surface $X$ and stable vector bundles on $X$ of degree zero was found out by M. S. Narasimhan and C. S. Seshadri in [16], their results have been extended to correspondences between representations of fundamental groups of various algebraic varieties and bundles with parabolic structure and with Higgs structure (and recently parabolic Higgs bundles). Stable parabolic bundles of parabolic degree zero correspond to irreducible unitary representations of the fundamental group of such a Riemann surface that a finite number of points are deleted from a compact Riemann surface. And stable Higgs bundles of degree zero correspond to irreducible (non-unitary) representations of the fundamental group of a compact Riemann surface. In the case of Higgs bundles, the results have been extended to higher dimensional cases. Moreover, in each case, the moduli of stable "bundles" had been constructed and proved that those moduli spaces are homeomorphic to those of the corresponding representations. In this paper, we shall give a definition of parabolic bundles in the higher dimensional cases and construct their moduli spaces.

In $\S 1$ we shall extend various notions on parabolic bundles on curves to that on non-singular projective varieties. We shall exploit C. T. Simpson's description of parabolic bundles on curves for our case. It leads us to simpler descriptions of parabolic homomorphisms, parabolic Hilbert polynomials, parabolic exact sequences than our original forms or Seshadri's. The moduli functor will be defined in this section. In $\S 2$ we shall deal with the boundedness of palabolic sheaves and the openness of the parabolic stability. C. T. Simpson's techniques on the estimation of dimensions of $H^{0}(E(m))$ for semi-stable sheaves $E$ enabled us to get a simpler proof of the "fundamental lemma" in [8]. $\S 3$ is devoted to constructing the moduli space. The method in this paper is a natural generalization 
of that used by U. N. Bhosle [2].

\section{Notation and Convention}

Let $f: X \rightarrow S$ be a smooth, projective, geometrically integral morphism of locally noetherian schemes, $E$ be a coherent $\mathcal{O}_{X}$-module and let $\mathcal{O}_{X}(1)$ be an $f$-very ample invertible sheaf. For a coherent $\mathcal{O}_{X}$-module $E$ and a numerical polynomial $H$, we denote simply by $Q u o t(E, H)$ the Quot-scheme $\operatorname{Quot}_{E / X / S}^{H}$. If $s$ is a geometric point of $S$, then $X_{s}$ means the geometric fiber of $X$ over $s$ and $E_{s}=E \otimes \mathcal{O}_{S} k(s)$. For a coherent $\mathcal{O}_{X}$-module $F$, the degree of $F$ with respect to $\mathcal{O}_{X}(1)$ is that of the first Chern class of $F$ with respect to $\mathcal{O}_{X_{s}}(1)=\mathcal{O}_{X}(1) \otimes \mathcal{O}_{X}$, and it is denoted by $\operatorname{deg}_{X(1)} F$ or simply $\operatorname{deg} F$. Moreover the rank of $F$ is denoted by $\operatorname{rk}(F), \mu(F)=\operatorname{deg} F / \operatorname{rk}(F)$ and $h^{i}(F)=\operatorname{dim}_{k(s)} H^{i}\left(X_{s}, F\right)$. For polynomials $f_{1}(n)$ and $f_{2}(n), f_{1}(n) \prec f_{2}(n)$ (or, $f_{1}(n) \preceq f_{2}(n)$ ) means that $f_{1}(n)<f_{2}(n)$ (or, $f_{1}(n) \leq f_{2}(n)$, resp.) for all sufficiently large integers $n$. For a polynomial $H$ and a number $m, H[m]$ denotes the polynomial such that $H[m](x)=H(m+x)$.

\section{Elementary Notions on Parabolic Sheaves}

Let $X$ be a non-singular, projective variety over an algebraically closed field $k$, $D \subset X$ be an effective Cartier divisor and let $\mathcal{O}_{X}(1)$ be a very ample invertible sheaf on $X$.

Definition 1.1 Let $E$ be a torsion free coherent $\mathcal{O}_{X}$-module. A quasi-parabolic structure ( with respect to $D$ ) is giving a filtration

$$
E=F_{1}(E) \supset F_{2}(E) \supset \cdots \supset F_{l}(E) \supset F_{l+1}(E)=E(-D),^{1}
$$

where $l$ is called the length of the filtration, $F_{i}(E) / F_{i+1}(E)$ is denoted by $G_{i}(E)$ and the Hilbert polynomial $\chi\left(G_{i}(E)(m)\right)$ is called the $i$-th multiplicity polynomial of $\left(E, F_{*}\right)$.

Definition 1.2 A quasi-parabolic sheaf $\left(E, F_{*}\right)$ is sajd to be a parabolic sheaf if it has a system of weights $\alpha_{1}, \alpha_{2}, \ldots, \alpha_{l}$ such that $0 \leq \alpha_{1}<\alpha_{2}<\cdots<\alpha_{l}<1$ where $l$ is the length of the filtration. The weight $\alpha_{i}$ is called the weight of the filter $F_{i}(E)$ and $\chi\left(G_{i}(m)\right)$ is called the mutiplicity polynomial of the weight $\alpha_{i}$.

Let $\left(E, F_{*}, \alpha_{*}\right)$ be a parabolic slieaf. For all real numbers $\alpha$, we put

$$
E_{\alpha}=F_{i}(E)(-[\alpha] D)
$$

\footnotetext{
${ }^{1} E(-D)$ is the Image $\left(E \otimes \mathcal{O}_{X}(-D) \rightarrow E\right)$
} 
if $\alpha_{i-1}<\alpha-[\alpha] \leq \alpha_{i}$ where $\alpha_{0}=\alpha_{l}-1$ and $\alpha_{l+1}=1$. In particular, we have $E_{\alpha_{i}}=F_{i}(E)$ for $i=1,2, \ldots, l+1$. Then we have the following filtration

$$
j_{*} j^{*} E=\bigcup_{m \in \mathbf{Z}} E(-m D)=\bigcup_{\alpha \in \mathbf{R}} E_{\alpha} \supset \cdots \supseteq E_{\alpha} \supseteq E_{\beta} \supseteq \cdots,
$$

where $j$ is the inclusion of $X-D$ to $X$. This filtration satisfies the following conditions:

1. If $\alpha \leq \beta$, then $E_{\alpha} \supseteq E_{\beta}$.

2. If $\varepsilon$ is a sufficiently small number, then $E_{\alpha-\epsilon}=E_{\alpha}$.

3. $E_{\alpha+1}=E_{\alpha}(-D)$ for all real numbers $\alpha$.

4. $E_{0}=E$.

5. The length of the filtration for $0 \leq \alpha \leq 1$ is finite.

Conversely, if $\bigcup_{m \in Z} E(-m D)$ has a filtration $\bigcup_{\alpha \in \mathbf{R}} E_{\alpha}$ which satisfies the above conditions, then $E$ has a unique parabolic structure giving the filtration. So we often denote a parabolic sheaf $\left(E, F_{*}, \alpha_{*}\right)$ by simply $E_{*}$.

Definition 1.4 Let $E_{\star}$ and $F_{\star}$ be two parabolic sheaves. An $\mathcal{O}_{X}$-homomorphism $f: E \rightarrow F$ is called a homomorphism of parabolic sheaves when the following condition is satisfied:

$$
f\left(E_{\alpha}\right) \subseteq F_{\alpha} \quad \text { for all } 0 \leq \alpha<1 .
$$

We denote by $\operatorname{Hom}^{\mathrm{par}}\left(E_{*}, F_{*}\right)$ the $k$-module consisting of all parabolic homomorphisms of $E_{m}$ to $F_{*}$ and by $\mathcal{H o m}^{\mathrm{par}}\left(E_{*}, F_{*}\right)$ the sheaf of all parabolic homomorphisms.

Definition 1.5 A parabolic sheaf $F_{*}$ is a parabolic subsheaf of a parabolic sheaf $E_{*}$ if the following conditions are satisfied:

1) $F$ is a subsheaf of $E$ and $E / F$ is torsion free.

2) $F_{\alpha} \subseteq E_{\alpha}$ for all $\alpha$.

3) If $F_{\alpha} \subseteq E_{\beta}$ for some $\beta>\alpha$, then $F_{\alpha}=F_{\beta}$.

Definition 1.6 A homomorphism $f: E_{\star} \rightarrow F_{*}$ of parabolic sheaves makes $F_{*}$ a quotient parabolic sheaf of $E_{*}$ if the following conditions are satisfied:

1) $f$ is surjective.

2) If $f\left(E_{\alpha}\right) \subseteq F_{\beta}$ for some $\beta>\alpha$, then $F_{\alpha}=F_{\beta}$.

Let $E_{*}$ be a parabolic sheaf and $F$ be a subsheaf of $E$ such that $E / F$ is torsion free. If we put $F_{\alpha}=E_{\alpha} \cap F$, then we get a parabolic subsheaf $F_{*}$ of $E_{*}{ }^{2}$ This parabolic structure of $F$ is the maximal one among all parabolic

\footnotetext{
${ }^{2}$ Since $E / F$ is torsion free, $E(-D) \cap F=F(-D)$.
} 
subsheaves of $E_{*}$ with the underlying subsheaf $F$. We call this structure of $F$ the induced sub-structure. Let $f$ be a surjective homomorphism of $E$ to a torsion free sheaf $G$. Put $G_{\alpha}=f\left(E_{\alpha}\right)$. Then we get a parabolic quotient sheaf $G_{*}$ of $E_{*}$. This parabolic structure of $G$ is the minimal one among all parabolic quotient sheaves of $E_{*}$ with the underlying quotient sheaf $f: E \rightarrow G$. We call this structure of $G$ the induced quotient structure.

Definition 1.7 Let $f: E_{*} \rightarrow F_{*}$ and $g: F_{*} \rightarrow G_{*}$ be two parabolic homomorphisms. The sequence

$$
0 \longrightarrow E_{*} \stackrel{f}{\longrightarrow} F_{*} \stackrel{g}{\longrightarrow} G_{*} \longrightarrow 0
$$

is said to be exact if for all real numbers $\alpha$ the sequence

$$
0 \longrightarrow E_{\alpha} \stackrel{f_{\alpha}}{\longrightarrow} F_{\alpha} \stackrel{g_{\alpha}}{\longrightarrow} G_{\alpha} \longrightarrow 0
$$

is exact.

Note that if the sequence (1.7.1) is exact, then $E_{*}$ and $G$. have the induced structures. Conversely, the sequence (1.7.1) is exact when $E_{*}$ and $G_{*}$ have the induced structures and the sequence

$$
0 \longrightarrow E \stackrel{f}{\longrightarrow} F \stackrel{g}{\longrightarrow} G \longrightarrow 0
$$

is exact.

Every torsion free sheaf $E$ has a natural parabolic structure:

$$
E=F_{1}(E) \subset F_{2}(E)=E(-D), \alpha_{1}=0 .
$$

This structure is called the special (parabolic) structure .

For a locally free sheaf $\mathcal{M}$ on $X, E * \otimes \mathcal{M}$ has a natural parabolic structure such that

$$
\left(E_{*} \otimes \mathcal{M}\right)_{\alpha}=E_{\alpha} \otimes \mathcal{M} .
$$

Hence, we regard $E_{*} \otimes \mathcal{M}$ as a parabolic sleaf with this structure.

Definition 1.8 1) The parabolic Euler characteristic of $E_{*}$ is

$$
\chi(E(-D))+\sum_{i=1}^{l} \alpha_{i} \chi\left(G_{i}\right),
$$

where $l$ is the length of the filtration. It is denoted by par- $\chi\left(E_{*}\right)$. The polynomial par- $\chi\left(E_{*}(m)\right)$ is called the parabolic Hilbert polynomial of $E_{*}$ and the polynomial par- $\chi\left(E_{*}(m)\right) / \operatorname{rk}(F)$ is denoted by par- $P_{E_{*}}(m)$. 
2) The parabolic degree of $E_{*}$ is

$$
\operatorname{deg} E+(n-1) ! \times\left\{\begin{array}{l}
\text { the coefficient of } m^{n-1} \text { of the polynomial } \\
\sum_{i=1}^{l} \alpha_{i} \chi\left(G_{i}(m)\right)
\end{array}\right\},
$$

where $n$ is the dimension of $X, r$ is the rank of $E$ and $\mathcal{O}_{X}{ }^{\oplus r}$. has the special structure The parabolic degree of $E_{*}$ is denoted by par-deg $E_{*}$ and we denote par-deg $E_{*} / \mathrm{rk}(E)$ by par- $\mu\left(E_{*}\right)$. The second term of the right side is denoted by $\operatorname{wt}\left(E_{*}\right)$.

It is easy to see that

$$
\operatorname{par}-\chi\left(E_{*}(m)\right)=\int_{0}^{1} \chi\left(E_{\alpha}(m)\right) d \alpha
$$

and

$$
\begin{aligned}
\operatorname{par}-\operatorname{deg}\left(E_{*}\right) & =(n-1) ! \times\left\{\begin{array}{c}
\text { the coefficient of } m^{n-1} \text { of the polynomial } \\
\left(\operatorname{par}-\chi\left(E_{*}(m)\right)-\operatorname{par}-\chi\left(\mathcal{O}_{X^{* r}}^{\oplus r}(m)\right)\right)
\end{array}\right\} \\
& =\int_{0}^{1} \operatorname{deg} E_{\alpha} d \alpha+r \cdot \operatorname{deg} D,
\end{aligned}
$$

where $r$ is the rank of $E$. Hence, if (1.7.1) is exact, then we have

$$
\begin{gathered}
\operatorname{par}-\chi\left(E_{*}(m)\right)+\operatorname{par}-\chi\left(G_{*}(m)\right)=\operatorname{par}-\chi\left(F_{*}(m)\right), \\
\operatorname{par}-\operatorname{deg}\left(E_{*}\right)+\operatorname{par}-\operatorname{deg}\left(G_{*}\right)=\operatorname{par}-\operatorname{deg}\left(F_{*}\right)
\end{gathered}
$$

and

$$
\operatorname{wt}\left(E_{*}\right)+\operatorname{wt}\left(G_{*}\right)=\operatorname{wt}\left(F_{*}\right) .
$$

Definition 1.10 1) $E_{*}$ is said to be parabolic stable (or, parabolic semi-stable) if for every parabolic subsheaf $F_{*}$ of $E_{\text {* with }} 0 \neq F \neq E$, we have

$$
\operatorname{par}-P_{F_{.}}(m) \prec \operatorname{par}-P_{E_{.}}(m) \quad \text { (or, } \preceq, \text { resp.). }
$$

2) $E_{*}$ is said to be parabolic $\mu$-stable (or, parabolic $\mu$-semi-stable) if for every parabolic subsheaf $F_{*}$ of $E_{*}$ with $0 \neq F \neq E$, we have

$$
\left.\operatorname{par}-\mu\left(F_{*}\right)<\operatorname{par}-\mu\left(E_{*}\right) \quad \text { (or, } \leq, \text { resp. }\right) .
$$

3) Let $e$ be an integer. $E$. is said to be of type $e$ if for every parabolic subsheaf $F_{*}$ of $E_{*}$ with $0 \neq F \neq E$, we have

$$
\operatorname{par}-\mu\left(F_{*}\right) \leq \operatorname{par}-\mu\left(E_{*}\right)+e .
$$


Remark 1.11 1. Let $f: E_{*} \rightarrow F_{*}$ be an injective parabolic homomorphism of parabolic sheaves with the same rank. Since $\int_{0}^{1} \chi\left(F_{\alpha} / E_{\alpha}(m)\right) d \alpha \succeq 0$, par- $P_{E .}(m) \preceq \operatorname{par}-P_{F_{0}}(m)$ and if the equality holds, then $f$ is an isomorphism of parabolic sheaves. Moreover, we have $\operatorname{par}-\mu\left(E_{*}\right) \leq \operatorname{par}-\mu\left(F_{*}\right)$. Hence, to verify the $(\mu-)$ stability (or $(\mu-)$ semi-stability) of $E_{*}$, it is enough to check the inequality for only parabolic subsheaves $F_{*}$ such that $E / F$ is torsion free and $F$. has the induced structure.

2. For a parabolic sheaf $E$. with the special structure, the parabolic Hilbert polynomial is not $\chi(E(m))$ but $\chi(E(-D)(m))$. So in general, the stability of a sheaf $E$ does not mean the parabolic stability of a parabolic sheaf $E$. with the special structure.

Every parabolic semi-stable sheaf has a Jordan-Hölder filtration. More precisely, every parabolic semi-stable sheaf $E_{*}$ has a filtration

$$
E=E^{0} \supset E^{1} \supset \cdots \supset E^{m}=0
$$

such that for all $i,\left(E^{i} / E^{i+1}\right)_{*}$ with the induced structure is parabolic stable and

$$
\text { par- } P_{E .}(m)=\operatorname{par}-P_{\left(E^{i} / E^{i+1}\right)_{\bullet}}(m) \text { for all } m \text {. }
$$

We denote by $\operatorname{gr}\left(E_{*}\right)$ the direct sum of parabolic sheaves $\oplus_{0}^{m-1}\left(E^{i} / E^{i+1}\right)$. It exists unique up to isomorphisms. The proof of this fact is given by the similar arguments as the case of semi-stable sheaves. (cf. [9]) Two parabolic sheaves $E_{*}$ and $F_{*}$ are said to be equivarent if $\operatorname{gr}\left(E_{*}\right)$ is isomorphic to $\operatorname{gr}\left(F_{*}\right)$.

Remark 1.12 We have two induced structures on $\left(E^{i} / E^{i+1}\right)$, i.e. the induced sub-structure from the induced quotient structure of $E / E^{i+1}$ and the induced quotient structure from the induced sub-structure of $E^{i}$. But it is easy to see that the two structures coincide.

Moreover every parabolic sheaf $E_{*}$ has the Harder-Narasimhan filtration, i.e. there exists the unique filtration

$$
E=E^{0} \supset E^{1} \supset \cdots \supset E^{m}=0
$$

such that all $\left(E^{i} / E^{i+1}\right)$ are parabolic $\mu$-semi-stable and for all $i$

$$
\operatorname{par}-\mu\left(\left(E^{i} / E^{i+1}\right)_{*}\right)<\operatorname{par}-\mu\left(\left(E^{i+1} / E^{i+2}\right)_{*}\right) .
$$

We can show this by a similar proof to that in the case of semi-stable sheaves. (cf. [21])

Let $f: X \rightarrow S$ be a smooth, projective, geometrically integral morphism of noetherian schemes, $D \subset X$ be a relative effective Cartier divisor with respect to $f$ and let $\mathcal{O}_{X}(1)$ be an $f$-very ample invertible sheaf. 
Definition 1.13 A coherent $\mathcal{O}_{X}$-module $E$ is said to be $f$-torsion free if it is flat over $S$ and for every geometric fiber $X_{s}$ of $f, E \otimes \mathcal{O}_{X}$, is a torsion free $\mathcal{O}_{X}$,-module.

Note that if $E$ is $f$-torsion free, then the canonical homomorphism

$$
E \otimes \mathcal{O}_{X}(-D) \rightarrow E
$$

is injective. In fact, if it is not injective, then $D$ contains an associated point $x$ of $E$ and the point $x$ is the associated point $E \otimes \mathcal{O}_{f^{-1}(f(x))}$ by the flatness of $E$ over $S$. The torsion freeness means that $x$ is the generic point of the fiber $f^{-1}(f(x))$. It contradicts the flatness of $D$.

Now we shall define the moduli functor for the family of parabolic semistable sheaves. Let $(S c h / S)$ be the category of locally noetherian schemes over $S$. Let $H$ and $H_{1}, H_{2}, \ldots H_{l}$ be polynomials and fix a set of real numbers $\alpha_{*}=$ $\left\{\alpha_{1}, \alpha_{2}, \ldots, \alpha_{l}\right\}$ such that $0 \leq \alpha_{1}<\alpha_{2}<\cdots<\alpha_{l}<1$.

Let $T$ be an object of $(S c h / S), E$ be a coherent $\mathcal{O}_{X_{T}}$-module, $F_{*}$ be a filtration of $E$ as in Definition 1.1 and $\alpha_{*}$ be a system of weights as in Definition 1.2. $\left(E, F_{*}, \alpha_{*}\right)$ is called a flat family of parabolic sheaves on $X_{T} / T$ if $E$ is $f_{T}$-torsion free and all $E / F_{i}(E)$ are flat over $T$ (hence, all $F_{i}$ are flat over $T$ ). Note that a flat family of parabolic sheaves has a filtration as in (1.3.1), hence we denote it simply by $E_{*}$.

Definition 1.14 For an object $T$ of $(S c h / S)$, set

$$
\operatorname{par}-\bar{\Sigma}_{D / X / S}^{H_{*}, \alpha *}(T)=\left\{\begin{array}{l}
E_{*} \\
\begin{array}{l}
E_{*} \text { is a flat family of parabolic sheaves } \\
\text { on } X_{T} / T \text { with the property (1.14.1) }
\end{array}
\end{array}\right\} / \sim
$$

where $\sim$ is the equivarence relation defined by (1.14.2).

(1.14.1) For every geometric point $t$ of $T,\left(E_{t}, F_{*, t}, \alpha_{*}\right)$ is parabolic semistable, $\chi\left(E_{t}(m)\right)=H(m)$ and $\chi\left(\left(E_{t} / F_{i+1}(E)_{t}\right)(m)\right)=H_{i}(m)$, where $F_{*, t}$ is the filtration :

$$
E_{t}=F_{1}(E)_{t} \supset F_{2}(E)_{t} \supset \cdots \supset F_{l+1}(E)_{t}=E_{t}(-D) .
$$

(1.14.2) $E_{*} \sim E_{*}^{\prime}$ if and only if there exist filtrations $E=E^{0} \supset E^{1} \supset$ $\cdots \supset E^{m}=0$ and $E^{\prime}=E^{\prime 0} \supset E^{\prime 1} \supset \cdots \supset E^{\prime m}=0$ such that for every geometric point $t$ of $T$, their restrictions to $X_{t}$ provide us with JordanHölder filtrations of $E_{t, *}$ and $E_{t, *}^{\prime}$, respectively, $\operatorname{gr}\left(E_{*}\right)=\bigoplus_{i=0}^{m} E^{i} / E^{i+1}$ is $T$-flat and that $\operatorname{gr}\left(E_{*}\right) \cong \operatorname{gr}\left(E_{*}^{\prime}\right) \otimes L$ for some invertible sheaf $L$ on $T$.

For a morphism $g: T^{\prime} \rightarrow T$ in $(\operatorname{Scl} / S), g^{*}$ defines a map of $\operatorname{par}-\bar{\Sigma}_{D / X}^{H_{*}, \alpha_{*}}(T)$ to $\operatorname{par}-\bar{\Sigma}_{D / X / S}^{H_{*}, \alpha *}\left(T^{\prime}\right)$. Then par- $\bar{\Sigma}_{D / X / S}^{H, \alpha *}$ is a contravariant functor of $(\mathrm{Sch} / S)$ to (Sets). We denote by par- $\Sigma_{D / X / S}^{H_{*}, \alpha \cdot}$ the sub-functor of par- $\bar{\Sigma}_{D / X / S}^{H_{*}, \alpha_{*}}$ consistiong of all flat families of parabolic stable sheaves. 


\section{Boundedness and Openness}

Let $f: X \rightarrow S$ be a smooth, projective, geometrically integral morphism of noetherian schemes, $D \subset X$ be a relative effective Cartier divisor with respect to $f$ and let $\mathcal{O}_{X}(1)$ be an $f$-very ample invertible sheaf. Assume $S$ is connected. We denote by $h$ the degree of $\mathcal{O}_{X}(1)$ on fibers. Fix polynomials $H$ and $H_{1}, H_{2}, \ldots H_{l}$ and a set of real numbers $\alpha_{*}=\left\{\alpha_{1}, \alpha_{2}, \ldots, \alpha_{l}\right\}$ such that $0 \leq \alpha_{1}<\alpha_{2}<\cdots<$ $\alpha_{l}<1$.

Let $\mathcal{F}\left(H, H_{*}, \alpha_{*}\right)$ be the family of classes of parabolic sheaves on the fibers of $X$ over $S$ such that $E_{*}$ is contained in $\mathcal{F}\left(H, H_{*}, \alpha_{*}\right)$ if and only if $E_{*}$ is a parabolic semi-stable sheaf on some geometric fiber of $X$ over $S, \chi(E(m))=$ $H(m), \chi\left(\left(E / F_{i+1}(E)\right)(m)\right)=H_{i}(m)$ and the system of weights is $\alpha_{*}$. For every member $E_{*}$ of $\mathcal{F}\left(H, H_{*}, \alpha_{*}\right)$, the parabolic Hilbert polynomial has the following description;

$$
\operatorname{par} \cdot \chi\left(E_{*}(m)\right)=H(m)-\sum_{i=1}^{l} \varepsilon_{i} H_{i}(m),
$$

where $\varepsilon_{i}=\alpha_{i+1}-\alpha_{i}\left(\right.$ set $\left.\alpha_{l+1}=1\right)$.

If $E_{*}$ is of type $e$, then $E$ is of type $e+\operatorname{deg} D$. In fact, we have

$$
\operatorname{wt}\left(E_{*}\right) \leq(n-1) ! \times\left(\begin{array}{l}
\text { the coefficient of } m^{n-1} \text { of } \\
\text { the polynomial } \chi\left(\left.E\right|_{D}(m)\right)
\end{array}\right)=\operatorname{rk}(E) \operatorname{deg} D .
$$

Hence, $\mu(F) \leq \operatorname{par}-\mu\left(F_{*}\right) \leq \operatorname{par}-\mu\left(E_{*}\right)+e \leq \mu(E)+\operatorname{deg} D+e$.

By the boundedness result on the family of coherent sheaves (cf. [10]), we have

Proposition 2.1 The family $\mathcal{F}\left(H, H_{*}, \alpha\right)$ is bounded if one of the following conditions is satisfied :

1) $S$ is a noetherian scheme over a field of characteristic zero.

2) The rank is not greater than 3 .

3) The dimension of $X$ over $S$ is not greater than 2 .

Definition 2.2 Let $e$ be a non-negative integer and let $E_{w}$ be a parabolic sheaf on some geometric fiber $X_{s}$.

1) $E$ is said to be of c-type $e$ if for general non-singular curves $C=$ $D_{1} \cdot D_{2} \cdots \cdots D_{n-1}, D_{i} \in\left|\mathcal{O}_{X}(1)\right|$, every subsheaf $E^{\prime}(\neq 0)$ of $E \otimes \mathcal{O}_{C}$ has a degree $\leq \operatorname{rk}\left(E^{\prime}\right)(\mu(E)+e)$.

2) $E_{*}$ is said to be parabolic e-stable (or, parabolic e-semi-stable) if $E_{*}$ is parabolic stable (or, parabolic semi-stable, resp.) and $E$ is of c-type $e$.

3) $E_{*}$ is said to be strictly parabolic e-semi-stable if it is e-semi-stable and if for every parabolic quotient sheaf $F_{*}$ of $E_{*}$ with par- $P_{E}=\operatorname{par}-P_{F_{*}}, F_{*}$ is parabolic $e$-semi-stable. 
Let $\mathcal{F}^{e}\left(H, H_{*}, \alpha_{*}\right)$ be the sub-family of $\mathcal{F}$ such that $E_{*}$ is contained in $\mathcal{F}^{e}\left(H, H_{*}, \alpha_{*}\right)$ if and only if $E_{*}$ is parabolic e-semi-stable.

By Lemma 3.3 in [8], we have

Proposition 2.3 The family $\mathcal{F}^{e}\left(H, H_{*}, \alpha_{*}\right)$ is bounded.

Let $\mu-\mathcal{F}(e, r)$ be the family of coherent sheaves on the fibers of $X$ over $S$ such that $E$ is contained in $\mu-\mathcal{F}(e, r)$ if and only if $E$ is $\mu$-semi-stable, $E$ is of c-type $e$ and $\operatorname{rk}(E) \leq r$. The following proposition is Corollary 2.5 in [22] for our case. (See for the proof, C. T. Simpson [22].)

Proposition 2.4 For each $\mu-\mathcal{F}(e, r)$, there exists an integer $B$ such that for all members $E$ of $\mu-\mathcal{F}(e, r)$, we have

$$
h^{0}(E(m)) \leq h \cdot \operatorname{rk}(E)(m+B+\mu(E) / h)^{n} / n ! \quad \text { for all } m \geq-B-\mu(E) / h,
$$

and $h^{0}(E(m))=0$ for all $m \leq-B-\mu(E) / h$.

In the case of stable sheaves, the following was proved by M. Maruyama [8] using the fundamental lemma 2.2 in [8]. C. T. Simpson got a simpler proof in the case of caracteristic zero using the Harder-Narasimhan filtration ([22]). In the parabolic case, we can also prove the following using the fundamental lemma 2.2 in [8]. Lemma 2.6 is equivalent to it. We give a simple proof using the technique of C.T.Simpson in [22].

Proposition 2.5 There exists an integer $N$ such that

1) if $E_{*} \in \mathcal{F}^{e}\left(H, H_{*}, \alpha_{*}\right)$ is parabolic stable, then for all $m \geq N$ and all parabolic subsheaves $F_{*}$ of $E_{*}$ with $0 \neq F \neq E$,

$$
\int_{0}^{1} h^{0}\left(F_{\alpha}(m)\right) d \alpha / \operatorname{rk}(F)<\int_{0}^{1} h^{0}\left(E_{\alpha}(m)\right) d \alpha / \operatorname{rk}(E),
$$

2) if $E_{*} \in \mathcal{F}^{e}\left(H, H_{*}, \alpha_{*}\right)$ is not parabolic stable, then for all $m \geq N$ and all parabolic subsheaves $F_{\text {* of }} E_{*}$ with $0 \neq F \neq E$,

$$
\int_{0}^{1} h^{0}\left(F_{\alpha}(m)\right) d \alpha / \operatorname{rk}(F) \leq \int_{0}^{1} h^{0}\left(E_{\alpha}(m)\right) d \alpha / \mathrm{rk}(E),
$$

and there exists a non-trivial parabolic subsheaf $E^{\prime}$ of $E_{*}$ such that for all $m \geq N$ and $i>0$,

$$
\int_{0}^{1} h^{0}\left(E_{\alpha}^{\prime}(m)\right) d \alpha / \mathrm{rk}\left(E^{\prime}\right)=\int_{0}^{1} h^{0}\left(E_{\alpha}(m)\right) d \alpha / \operatorname{rk}(E)
$$

$h^{i}\left(E^{\prime}(m)\right)=0$ and $E^{\prime}(m)$ is generated by its global sections. 
Lemma 2.6 (Fundamental lemma 2.2 in [8]) Let $S$ be a locally noetherian, connected scheme, $f: X \rightarrow S$ be a smooth, projective, geometrically integral morphism of relative dimension $n$ and let $\mathcal{O}_{X}(1)$ be an $f$-very ample invertible sheaf on $X$. Let a be a rational number, $r$ be a positive integer and $P(m)$ be $a$ polynomial of degree $n$ with the highest term $h m^{n} / n$ ! where $h$ is the degree of $\mathcal{O}_{X}(1)$ on fibers of $f$. Then there exist integers $L$ and $M$ such that if $F$ is a torsion free coherent $\mathcal{O}_{X}$,-module of rank $r^{\prime} \leq r$ for some geometric point $s$ of $S$ and if $F$ has the properties;

1) for general non-singular curves $C=D_{1} \cdot D_{2} \cdots D_{n-1}, D_{i} \in\left|\mathcal{O}_{X},(1)\right|$, every coherent subsheaf $E(\neq 0)$ of $F \otimes \mathcal{O}_{C}$ has a degree $\leq \operatorname{rk}(E) a$,

2) $\mu(F) \leq M$,

then for all $m \geq L$, the following inequality holds;

$$
h^{0}(F(m)) \leq r^{\prime} P(m)
$$

To prove Lemma 2.6, we need the following lemma

Lemma 2.7 Assume that $u$ and a are rational numbers with $u \leq a$ and $r$ is a positive integer. Let $\mathcal{F}(a, u, r)$ be the family of classes of coherent sheaves on fibers of $X$ over $S$ such that $F$ is contained in $\mathcal{F}(a, u, r)$ if and only if $F$ is torsion free, $\operatorname{rk}(F) \leq r, \mu(F) \leq u$ and $F$ satisfies the condition 1) in Lemma 2.6. Then there exist an integer $B$, a non-negative number $C$ and a polynomial $\Phi(x)$ of degree $\leq n-2$ such that

$h^{0}(F(m)) \leq \frac{h \cdot \operatorname{rk}(F)}{n !}\left(m+B+\frac{u+(\operatorname{rk}(F)-1) a}{h \cdot \operatorname{rk}(F)}\right)^{n}+\Phi(m) \quad$ if $m+B \geq-u / h$, if $-a / h \leq m+B \leq-u / h, h^{0}(F(m)) \leq C$ and if $m+B \leq-a / h, h^{0}(F(m))=0$, $B$ does not depend on $u$ and $\Phi(x)$ has the property;

$$
\text { for all } x \geq-B-u / h, \quad \Phi^{\prime}(x) \geq 0 \quad \text { and } \quad \Phi(-B-u / h) \geq 0 .
$$

Proof. We prove the assertion by an induction on $n$. In the case of $n=1$, apply Proposition 2.4 to the case $\mu-\mathcal{F}(0, r)$, then we have an integer $B_{1}$ depending only on $X, \mathcal{O}_{X}(1)$ and $r$ such that for all $\mu$-semi-stable sheaves $E$ of rank $\leq r$, we have

$$
h^{0}(E(m)) \leq h \cdot \operatorname{rk}(E)\left(m+B_{1}+\frac{\mu(E)}{h}\right) \quad \text { if } m+B_{1} \geq-\mu(E) / h,
$$

and $h^{0}(E(m))=0$ if $m+B_{1} \leq-\mu(E) / h$.

Let $F=F^{1} \supset F^{2} \supset \cdots F^{t} \supset 0$ be the Harder-Narasimhan filtration of $F$ and let $Q^{i}=F^{i} / F^{i+1}$. Then we have $\mu\left(Q^{1}\right) \leq \mu(F) \leq u$ and by the assumption 
1) in Lemma 2.6, $\mu\left(Q^{i}\right) \leq \mu\left(Q^{t}\right) \leq a$. Since all $Q^{i}$ are in $\mu-\mathcal{F}(0, r)$, we have

$$
\begin{aligned}
h^{0}(F(m)) \leq & \sum_{i} h^{0}\left(Q^{i}(m)\right) \\
\leq & \sum_{i} h \cdot \operatorname{rk}\left(Q^{i}\right) \max \left\{\left(m+B+\frac{\mu\left(Q^{i}\right)}{h}\right), 0\right\} \\
\leq & h(\operatorname{rk}(F)-1) \max \left\{\left(m+B+\frac{a}{h}\right), 0\right\} \\
& +h \cdot \max \left\{\left(m+B+\frac{u}{h}\right), 0\right\} .
\end{aligned}
$$

This is the desired one in the case of $n=1$.

Let $H$ be a general hyperplane in $\left|\mathcal{O}_{X_{\theta}}(1)\right|$. By the exact sequence

$$
\left.0 \longrightarrow F(m-1) \longrightarrow F(m) \longrightarrow F\right|_{H}(m) \longrightarrow 0
$$

and the induction hypothesis, there exist $B_{n-1}, C_{n-1}$ and $\Phi_{n-1}(x)$ such that if $m+B_{n-1} \geq-u / h$,

$$
\begin{aligned}
& h^{0}(F(m))-h^{0}(F(m-1)) \leq \\
& \quad \frac{h \cdot \operatorname{rk}(F)}{(n-1) !}\left(m+B_{n-1}+\frac{u+(\operatorname{rk}(F)-1) a}{h \cdot \operatorname{rk}(F)}\right)^{n-1}+\Phi_{n-1}(m),
\end{aligned}
$$

if $-a / h \leq m+B_{n-1} \leq-u / h, h^{0}(F(m))-h^{0}(F(m-1)) \leq C_{n-1}$ and if $m+B_{n-1} \leq$ $-a / h, h^{0}(F(m))=h^{0}(F(m-1))$. Set $B_{n}=B_{n-1}+1$ and $C_{n}=C_{n-1}\left(\frac{a-u}{h}\right)$. Then, if $m+B_{n} \leq-a / h$, we have $h^{0}(F(m))=0$ and if $-a / h \leq m+B_{n} \leq-u / h$, we have $h^{0}(F(m)) \leq C_{n}$. Moreover, if $m+B_{n} \geq-u / h$, then we have

$$
\begin{aligned}
& h^{0}(F(m)) \leq C_{n}+ \\
& \quad \int_{-B_{n-1}-u / h}^{m+1}\left(\frac{h \cdot \operatorname{rk}(F)}{(n-1) !}\left(x+B_{n-1}+\frac{u+(\operatorname{rk}(F)-1) a}{h \cdot \operatorname{rk}(F)}\right)^{n-1}+\Phi_{n-1}(x)\right) d x .
\end{aligned}
$$

We can take a polynomial $\Phi_{n}(x)$ so that $\Phi_{n}^{\prime}(x)=\Phi(x+1), \Phi_{n}\left(-B_{n}-u / h\right) \geq 0$ and if $m+B_{n} \geq-u / h$, the right-hand side of the above inequality is smaller than

$$
\frac{h \cdot \operatorname{rk}(F)}{n !}\left(m+B_{n}+\frac{u+(\operatorname{rk}(F)-1) a}{h \cdot \operatorname{rk}(F)}\right)^{n}+\Phi_{n}(m) .
$$

Then $B_{n}, C_{n}$ and $\Phi_{n}(x)$ satisfy the desired conditions.

Proof of Lemma 2.6. Let $P(m)=h \cdot m^{n} / n !+a_{1} m^{n-1}+($ terms of degree $\leq$ $n-2)$ and $u$ be a rational number such that

$$
h\left(B+\frac{u+\left(r^{\prime}-1\right) a}{h r^{\prime}}\right)<a_{1} \quad \text { for all positive integers } r^{\prime} \leq r .
$$


Then there exists an integer $L \geq-B-u / h$ such that for all $m \geq L$ and $0 \leq r^{\prime} \leq r$, we have

$$
\frac{h}{n !}\left(m+B+\frac{u+\left(r^{\prime}-1\right) a}{h r^{\prime}}\right)^{n}+\Phi(m)<P(m) .
$$

Then for $M=u$ and $L$, the assertion of Lemma 2.6 holds.

Proof of Proposition 2.5. By the boundedness of $\mathcal{F}^{\mathrm{e}}\left(H, H_{*}, \alpha_{*}\right)$, there exists an integer $N_{1}$ such that for all $E_{*} \in \mathcal{F}^{e}\left(H, H_{*}, \alpha_{*}\right), i>0, m \geq N_{1}$ and $0 \leq \alpha \leq 1$, we have $h^{i}\left(E_{\alpha}(m)\right)=0$. Hence $\int_{0}^{1} h^{0}\left(E_{\alpha}(m)\right) d \alpha / \operatorname{rk}(E)=$ par- $P_{E_{*}}(m)$. Let us apply Lemma 2.6 to the case $P(m)=$ par- $P_{E .}(m), r=\operatorname{rk}(E)$ and $a=\mu(E)+e$ where $E_{*}$ is a member of $\mathcal{F}^{e}\left(H, H_{*}, \alpha_{*}\right)$. Note that these don't depend on choices of $E_{*}$. Then we obtain integers $L$ and $M$ satisfying the conditions in Lemma 2.6. We may assume that $L \geq N_{1}$. Let $F$ be a coherent subsheaf of $E$. Since $E$ satisfies the condition 1) in Lemma 2.6, for all $\alpha$ with $0 \leq \alpha \leq 1$, the subsheaf $F_{\alpha}=E_{\alpha} \cap F$ of $E$ also satisfies it. If $\mu(F) \leq M$ (hence, $\mu\left(F_{\alpha}\right) \leq M$ ), then we have

$$
h^{0}\left(F_{\alpha}(m)\right)<\operatorname{rk}(F) P(m) \text { for all } m \geq L .
$$

Therefore we have

$$
\int_{0}^{1} h^{0}\left(F_{\alpha}(m)\right) d \alpha / \operatorname{rk}(F)<P(m) \text { for all } m \geq L .
$$

Let $\mathcal{G}$ be the family of classes of parabolic sheaves on the fibers of $X$ over $S$ such that $F_{*}$ is contained in $\mathcal{G}$ if and only if for some member $E_{*}$ of $\mathcal{F}^{e}\left(H, H_{*}, \alpha_{*}\right)$, $F_{*}$ is a parabolic subsheaf of $E$. such that $E / F$ is torsion free, $\mu(F) \geq M$ and $F_{*}$ has the induced structure. Since $\mathcal{F}^{e}\left(H, H_{*}, \alpha_{*}\right)$ is bounded, $\mathcal{G}$ is bounded by Corollary 1.5 .1 in [11]. Therefore there exists an integer $L^{\prime} \geq L$ such that for all $F_{*} \in \mathcal{G}, i>0, m \geq L^{\prime}$ and $0 \leq \alpha \leq 1$, we have $h^{i}\left(F_{\alpha}(m)\right)=0$ and $F(m)$ is generated by its global sections. The set of all parabolic Hilbert polynomials of $F_{*}$ with $F_{*} \in \mathcal{G}$ is a finite set. Hence, there exists an integer $N \geq L^{\prime}$ such that for all $F_{*} \in \mathcal{G}$ and $m \geq N$,

$$
\int_{0}^{1} h^{0}\left(F_{\alpha}(m)\right) d \alpha / \mathrm{rk}(F)=\operatorname{par}-P_{F_{*}}(m) \leq P(m)
$$

and moreover if $F_{*}$ is a parabolic subsheaf of a parabolic stable sheaf $E_{*} \in$ $\mathcal{F}^{e}\left(H, H_{*}, \alpha_{*}\right)$, we have

$$
\int_{0}^{1} h^{0}\left(F_{\alpha}(m)\right) d \alpha / \mathrm{rk}(F)<P(m) .
$$

If $F_{*}$ is a parabolic subsheaf of $E_{*} \in \mathcal{F}^{e}\left(H, H_{*}, \alpha_{*}\right)$ and $\mu(F) \geq M$, then there exist $F^{\prime}, \in \mathcal{G}$ and an injective parabolic homomorphism of $F_{*}$ to $F^{\prime}{ }_{*}$. Then we have $\int_{0}^{1} h^{0}\left(F_{\alpha}(m)\right) d \alpha / \mathrm{rk}(F) \leq \int_{0}^{1} h^{0}\left(F_{\alpha}^{\prime}(m)\right) d \alpha / \mathrm{rk}\left(F^{\prime}\right) \leq P(m)$. Hence, 
the assertion 1) and the previous part of the assertion 2) was proved. If $E_{*} \in$ $\mathcal{F}^{e}\left(H, H_{*}, \alpha_{*}\right)$ is not stable, then there exists a non-trivial parabolic subsheaf $E^{\prime}$. of $E_{*}$ such that par- $P_{E_{*}^{\prime}}=$ par- $P_{E_{*}}$. For such $E^{\prime}$, we have $\mu\left(E^{\prime}\right) \geq$ $\operatorname{par}-\mu(E)-\operatorname{deg} D$. We may assume that $\operatorname{par}-\mu(E)-\operatorname{deg} D \geq M$. Hence, $E^{\prime}$. must be contained in $\mathcal{G}$. Therefore, we have $\int_{0}^{1} h^{0}\left(E_{\alpha}^{\prime}(m)\right) d \alpha / \mathrm{rk}\left(E^{\prime}\right)=$ $\int_{0}^{1} h^{0}\left(E_{\alpha}(m)\right) d \alpha / \mathrm{rk}(E)$ and $E^{\prime}(m)$ is generated by its global sections.

Strictly parabolic e-semi-stablility is an open property. In fact, we have:

Proposition 2.8 Let $g: Y \rightarrow T$ be a smooth, projective, geometrically integral morphism of locally noetherian schemes, $\mathcal{O}_{Y}(1)$ be a g-very ample invertible sheaf, $D \subset Y$ be a relative effective Cartier divisor and $\left(E, F_{*}, \alpha_{*}\right)$ be a flat familly of parabolic sheaves on $Y / T$. If $H^{i}\left(Y_{t}, \mathcal{O}_{Y}(1) \otimes k(t)\right)=0$ for all $i>0$ and $t \in T$, then there exist open sets $T^{s s}$ and $T^{s}$ of $T$ such that for all algebraically closed fields $k$,

$$
\begin{aligned}
T^{s s}(k) & =\left\{t \in T(k) \mid\left(E, F_{*}, \alpha_{*}\right) \otimes k(t) \text { is strictly parabolic e-semi-stable }\right\} \\
T^{s}(k) & =\left\{t \in T(k) \mid\left(E, F_{*}, \alpha_{*}\right) \otimes k(t) \text { is parabolic e-stable }\right\} .
\end{aligned}
$$

Proof. First, we shall prove that the property that a parabolic sheaf is of type $e$ (or, parabolic semi-stable or, parabolic stable, resp.) is open. Before prove the above, we shall prepare some lemmas.

Lemma 2.9 Let $X \rightarrow S$ be a projective morphism of locally noetherian schemes, $\mathcal{O}_{X}(1)$ be an $f$-very ample invertible sheaf, $\phi: E \rightarrow F$ be an $\mathcal{O}_{X}$-homomorphism of coherent $\mathcal{O}_{X}$-modules flat over $S$. Then for all polynomials $P(m)$, the following subset of $S$ is closed :

$$
Z(P)=\left\{s \in S \mid \chi\left(\operatorname{ker}\left(\phi_{s}\right)(m)\right) \succeq P(m)\right\}
$$

Moreover, if for all points $s \in S, \operatorname{rk}\left(\operatorname{ker}\left(\phi_{s}\right)\right)=r$, then

$$
W(a)=\left\{s \in S \mid \operatorname{deg}\left(\operatorname{ker}\left(\phi_{s}\right)\right) \geq a\right\} .
$$

is closed.

Proof. We may assume that $S$ is connected and noetherian. Set $C=F / \phi(E)$. For sufficiently large integer $m$ and all points $s$ of $S$, we have

$$
h^{0}\left(\left.C(m)\right|_{X_{s}}\right)=\operatorname{dim} f_{*}(C(m)) \otimes k(s) .
$$

Hence, for sufficiently large $m$, the function $h^{0}\left(\left.C(m)\right|_{X}\right)$ is upper semicontinuous and since $h^{0}\left(\left.F(m)\right|_{X}\right)$ is constant on $S$, the function $h^{0}\left(\left(\phi(m)_{s}\right)(E(m))\right)$ is lower semicontinuous. Moreover, since $h^{0}\left(\left.E(m)\right|_{X}\right)$ is constant on $S$, the function $h^{0}\left(\operatorname{ker}\left(\phi(m)_{s}\right)\right)$ is upper semicontinuous. Obviously, $\operatorname{ker}\left(\phi(m)_{s}\right)=\operatorname{ker}\left(\phi_{s}\right)(m)$. 
If we choose a large $M$ such that for all points $s$ of $S, h^{0}\left(\operatorname{ker}\left(\phi_{s}\right)(M)\right)=$ $\chi\left(\operatorname{ker}\left(\phi_{s}\right)(M)\right)$ and $\chi\left(\operatorname{ker}\left(\phi_{s}\right)(m)\right) \succeq P(m)$ if and only if $\chi\left(\operatorname{ker}\left(\phi_{s}\right)(M)\right) \geq$ $P(M)$, by the upper semi-continuity, we know that $Z(P)$ is closed.

Since the degree of a coherent sheaf $K$ on $X$, of rank $r$ is $(n-1) ! \times$ (the coefficient of $m^{n-1}$ of the polynomial $\left.\chi(K(m))-r \cdot \chi\left(\mathcal{O}_{X_{s}}(m)\right)\right)$, the closedness of $W(a)$ is the immediate result of that of $Z(P)$.

The following lemma is an easy exercise, hence, we leave the proof to the reader.

Lemma 2.10 Let $\left\{f_{1}, f_{2}, \ldots f_{l}\right\}$ be a finite set of upper semi-continuous functions on a topological space $T$ such that for all $i$ and integers $N$, the set $f_{i}(T) \cap$ $\{r \in \mathrm{R} \mid r \leq N\}$ is finite. Then the function $\sum_{i=1}^{l} f_{i}$ is upper semi-continuous.

We may assume that $T$ is connected. Let $\mathcal{G}$ be the family of coherent sheaves on fibers of $Y$ over $T$ such that $F$ is contained in $\mathcal{G}$ if and only if $F$ is a subsheaf of $E_{t}$ such that $E_{t} / F$ is torsion free and $\operatorname{deg} F>\operatorname{rk}(F)\left(\mu\left(E_{t}\right)-\operatorname{deg} D+e\right)$ where $\mathrm{t}$ is a point such that $F$ is on $Y_{t}$. By Corollary 1.5.1 in [11], $\mathcal{G}$ is bounded. Let $\Theta$ be the set of all Hilbert polynomials of members of $\mathcal{G}$. Then $\Theta$ is a finite set.

For each member $\theta$ of $\Theta$, let $Q_{\theta}$ be the quot-scheme Quot $(E, H-\theta)$ where $H$ is the Hilbert polynomial of $E$ on fibers. The universal quotient on $Y_{Q_{\theta}}$ defines the exact sequence:

$$
0 \longrightarrow \tilde{F} \longrightarrow E \otimes \mathcal{O}_{Q} \longrightarrow \tilde{G} \longrightarrow 0 \text {. }
$$

For every $\alpha$ such that $0 \leq \alpha \leq 1$, let $\phi_{\alpha}$ be the canonical homomorphicm of $\tilde{F}$ to $E / E_{\alpha} \otimes \mathcal{O}_{Q_{\theta}}$. Then by virtue of Lemma 2.9 , the function $\operatorname{deg}\left(\left.\tilde{F}\right|_{Y_{q}}\right)_{\alpha}$ is an upper semi-continuous function on $Q_{\theta}$. Moreover by Lemma 2.10 , the function $\operatorname{par}-\operatorname{deg}\left(\left.\tilde{F}\right|_{Y_{q}}\right)_{*}$ is also upper semi-continuous. Hence, the set

$$
Z_{\theta}=\left\{q \in Q_{\theta} \mid \operatorname{par}-\mu\left(\left(\left.\tilde{F}\right|_{Y_{q}}\right)_{*}\right)>\operatorname{par}-\mu\left(\left.E_{*}\right|_{Y_{q}}\right)+e\right\}
$$

is a closed subset of $Q_{\theta}$. Set

$$
U=T-\bigcup_{\theta \in \Theta} \pi_{\theta}\left(Z_{\theta}\right)
$$

where $\pi_{\theta}$ is the projection of $Q_{\theta}$ to $T$. Then $U$ is an open subset of $T$ and it is the set of all points $t$ of $T$ such that $\left.E_{*}\right|_{Y_{t}}$ is of type $e$. In fact, for all points $q$ of $Z_{\theta},\left.E_{*}\right|_{Y_{q}}$ is not of type $e$. And if $t_{0}$ be a point such that $\left.E_{*}\right|_{Y_{i_{0}}}$ is not of type $e$, then there exists a subsheaf $F$ of $E_{t_{0}}$ such that $E_{t_{0}} / F$ is torsion free and $\operatorname{par}-\mu\left(F_{*}\right)>\operatorname{par}-\mu\left(\left.E_{*}\right|_{Y_{t_{0}}}\right)+e$, (hence, $\mu(F)>\mu(E)-\operatorname{deg} D+e$ ), where $F_{*}$ has the induced structure. So, $F$ is contained in $\mathcal{G}$. Therefore $t_{0}$ is in $\pi_{\theta}\left(Z_{\theta}\right)$ where $\theta$ is the Hilbert polynomial of $F$.

The proof of the openness of the property that a parabolic sheaf is parabolic semi-stable (or, parabolic stable, resp.) is similar to the above if we use the 
(parabolic) Hilbert polynomials instead of (parabolic) degrees. Note that by Lemma 2.9 and 2.10 , for large integer $m$, the function par- $\chi\left(\left(\left.\tilde{F}\right|_{Y_{q}}\right)_{*}(m)\right)$ is upper semi-continuous on $Q_{\theta}$ when $Q_{\theta}$ and $\tilde{F}$ defined as above.

Now by a similar proof as in Lemma 3.5 of [8], we see that the property that a parabolic sheaf is parabolic $e$-semi-stable (or, parabolic $e$-stable, resp.) is open. Moreover, the proof of this Proposition 2.8 is similar to that of Lemma 3.6 in [9] if we use the technic as above. Hence, we omit the rest of the proof.

Remark 2.11 By the above proof, we know that the property that a parabolic sheaf is parabolic e-stable is open. Hence, $\operatorname{par}_{-} \Sigma_{D / X / S}^{H_{*}, \alpha_{\bullet}, e}$ (or, par- $\bar{\Sigma}_{D / X / S}^{H_{*}, \alpha_{*}, e}$, resp.) is an open subfunctor of par- $\Sigma_{D / X / S}^{H_{*}, \alpha_{*}}$ (or, $\operatorname{par}-\bar{\Sigma}_{D / X / S}^{H_{*}, \alpha *}$, resp.) consisting of all flat families of parabolic $e$-stable (or, strictly parabolic $e$-semi-stable, resp.) sheaves.

\section{Construction of Moduli Space}

From now on, we shall fix the following situation:

(3.0) Let $S$ be a scheme of finite type over a universally Japanese ring $\Xi$ and let $f: X \rightarrow S$ be a smooth, projective, geometrically integral morphism such that the dimension of each fiber of $X$ over $S$ is $n$. Let $\mathcal{O}_{X}(1)$ be an $f$-very ample invertible sheaf such that for all points $s$ in $S$ and $i>0, H^{i}\left(X_{s}, \mathcal{O}_{X}(1) \otimes \mathcal{O}_{X_{s}}\right)=$ 0 . Let $D \subset X$ be a relative effective Cartier divisor.

Fix a non-empty family $\mathcal{F}^{\mathrm{e}}\left(H, H_{*}, \alpha_{*}\right)$. By the boundedness of $\mathcal{F}^{\mathrm{e}}\left(H, H_{*}, \alpha_{*}\right)$, there exists an integer $N_{0}$ such that for every member $E_{*}$ of $\mathcal{F}^{e}\left(H, H_{*}, \alpha_{*}\right)$, the conditions 1), 2) in Proposition 2.5 and the following conditions are satisfied:

(3.0.1) For all $i$ and $m \geq N_{0}, F_{i}(E)(m)$ and $\left(E / F_{i}(E)\right)(m)$ are generated by its global sections.

(3.0.2) $\quad H^{j}\left(F_{i}(E)(m)\right)=0$ and $H^{j}\left(\left(E / F_{i}(E)\right)(m)\right)=0 \quad$ for all $j \geq 1, i$ and $m \geq N_{0}$.

Fix an integer $m \geq N_{0}$ and let $V_{m}$ be a free $\Xi$-module of rank $H(m)$. Let $Q$ be the Quot-scheme $\operatorname{Quot}\left(V_{m} \otimes \mathcal{O}_{X}, H[m]\right)$ and let $V_{m} \otimes \mathcal{O}_{X_{Q}} \rightarrow \tilde{E}(m)$ be the universal quotient. Moreover, let $Q_{i}$ be the Quot-scheme Quot $\left(V_{m} \otimes \mathcal{O}_{X}, H_{i}[m]\right)$ and let $V_{m} \otimes \mathcal{O}_{X_{Q_{i}}} \rightarrow \widetilde{E}_{i}(m)$ be the universal quotient for $i=1, \ldots, l$.

Let $\nu: Q \rightarrow \mathrm{Pic}_{X / S}$ be the morphism determined by the invertible sheaf $\operatorname{det} \tilde{E}(m)$ (cf. [8]) and let $P$ be a finite union of connected components which contain $\nu(Q)$. Then we have a $P$-morphism $\tau$ of $Q$ to the Gieseker space $Z$ defined in [8]. $Z / P$ is the $\mathrm{P}^{N}$-bundle in étale topology and for a $K$-valued 
geometric point $x$ of $P$, a fiber $Z_{x}$ is $\mathrm{P}\left(\operatorname{Hom}\left(\wedge^{r} V_{m} \otimes k(x), H^{0}\left(L_{x}\right)\right)^{\vee}\right)$ where $L_{x}$ is the invertible sheaf corresponding to $x$ and $r$ is the rank of $\widetilde{E}$ on fibers. (For details, see $\S 4$ in [8].)

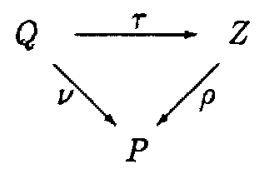

We shall define a sequence of morphisms

$$
R_{1} \rightarrow R_{2} \rightarrow \cdots \rightarrow R_{l} \rightarrow Q,
$$

coherent $\mathcal{O}_{X_{R_{i}}}$-module $\vec{E}_{i}(m)$ and surjections $\vec{E}_{i+1}(m) \otimes \mathcal{O}_{X_{R_{i}}} \rightarrow \bar{E}_{i}(m)$, inductively. Let $R_{l}$ be the Quot-scheme Quot $\left(\tilde{E}(m), H_{l}[m]\right)$ and let $\tilde{E}(m) \otimes \mathcal{O}_{X_{R_{l}}} \rightarrow$ $\bar{E}_{l}(m)$ be the universal quotient. Assume that those are defined for $j \geq i+1$. Let $R_{i}$ be the Quot-scheme Quot $\left(\bar{E}_{i+1}(m), H_{i}[m]\right)$ and let $\bar{E}_{i+1}(m) \otimes \mathcal{O}_{X_{R_{i}}} \rightarrow \bar{E}_{i}(m)$ be the universal quotient.

Then we have the sequence of surjections of $\mathcal{O}_{X_{R_{1}}}$-modules which are flat over $R_{1}$.

$$
V_{m} \otimes \mathcal{O}_{X_{R_{1}}} \stackrel{\phi}{\longrightarrow} \tilde{E}(m) \otimes \mathcal{O}_{X_{R_{1}}} \stackrel{\phi_{l}}{\longrightarrow} \bar{E}_{l}(m) \otimes \mathcal{O}_{X_{R_{1}}} \stackrel{\phi_{l-1}}{\longrightarrow} \cdots \stackrel{\phi_{1}}{\longrightarrow} \bar{E}_{1}(m) .
$$

Note that $R_{i}$ is a closed subscheme of $Q \times \prod_{j=i}^{l} Q_{j}$. (Use the lemma 4.3 in [23].) Let $\phi_{l}^{\prime}: \tilde{E}(m) \otimes \mathcal{O}_{X_{R_{1}}}(-D) \rightarrow \bar{E}_{l}(m) \otimes \mathcal{O}_{X_{R_{1}}}$ be the restriction of $\phi_{l}$ to $\widetilde{E}(m) \otimes \mathcal{O}_{X_{R_{1}}}(-D)$. By the lemma 4.3 in [23], there exists a unique closed subscheme $\bar{\Gamma}$ of $R_{1}$ such that for all morphisms $g: T \rightarrow R_{1}, g^{*}\left(\phi_{l}^{\prime}\right)=0$ if and only if $g$ factors through $\tilde{\Gamma}$. Let $Q^{0}$ be an open subscheme of $Q$ such that for every algebraically closed field $K, Q^{0}(K)=\{x \in Q(K) \mid \tilde{E}(m) \otimes k(x)$ is torsion free $\}$ (cf.[11]). Note that the restriction of $\nu$ to $Q^{0}$ is an immersion (see Proposition 4.9 in [8]). Set $R_{i}^{0}=R_{i} \times_{Q} Q^{0}$ and $\Gamma^{0}=\bar{\Gamma} \times{ }_{Q} Q^{0}$.

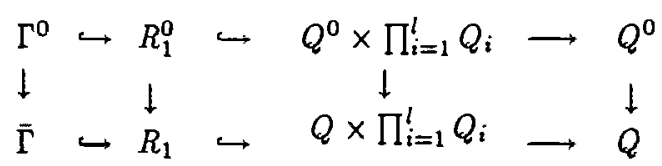

On $X_{\Gamma^{0}}$, we have the sequence of surjections:

$$
V_{m} \otimes \mathcal{O}_{X_{\Gamma^{0}}} \stackrel{\phi}{\longrightarrow} \tilde{E}(m) \otimes \mathcal{O}_{X_{\Gamma^{0}}} \stackrel{\phi_{l}}{\longrightarrow} \bar{E}_{l}(m) \otimes \mathcal{O}_{X_{\Gamma^{0}}} \stackrel{\phi_{l-1}}{\longrightarrow} \cdots \stackrel{\phi_{1}}{\longrightarrow} \tilde{E}_{1}(m) \otimes \mathcal{O}_{X_{\Gamma^{0}}}
$$

Let $\bar{F}_{i+1}(\tilde{E}(m))$ be the kernel of the homomorphism

$$
\phi_{i} \circ \phi_{i-1} \circ \cdots \circ \phi_{l}: \tilde{E}(m) \otimes \mathcal{O}_{X_{\mathrm{r}^{0}}} \longrightarrow \bar{E}_{i}(m) \otimes \mathcal{O}_{X_{\mathrm{r}^{0}}}
$$


for $i=1, \ldots, l$ and let $\bar{F}_{1}(\tilde{E}(m))=\tilde{E}(m) \otimes \mathcal{O}_{X_{\Gamma^{0}}}$. Since $\vec{F}_{1}(\tilde{E}(m))$ is $\left(f \times 1_{\Gamma^{0}}\right)$ torsion free, the homomorphism $\widetilde{E}(m) \otimes \mathcal{O}_{X_{\mathrm{r} 0}}(-D) \rightarrow \widetilde{E}(m) \otimes \mathcal{O}_{X_{\mathrm{r}^{0}}}$ is injective and hence, $\bar{F}_{l+1}(\tilde{E}(m))=\tilde{E}(m) \otimes \mathcal{O}_{X_{\Gamma^{0}}}(-D)$. Then $\left(\tilde{E}(m) \otimes \mathcal{O}_{X_{\Gamma^{0}}}, \bar{F}_{*}, \alpha_{*}\right)$ is a flat family of parabolic sheaves on $X_{\Gamma^{0}}$ over $\Gamma^{0}$.

Let $U_{i}$ be the maximal open subscheme of $Q_{i}$ such that for all points $x$ of $U_{i}$ and $j \geq 1, H^{j}\left(\tilde{E}_{i}(m) \otimes k(x)\right)=0$ and $V_{m} \otimes \mathcal{O}_{U_{i}} \longrightarrow f_{i m}\left(\tilde{E}_{i}(m) \otimes \mathcal{O}_{X_{V_{i}}}\right)$ is surjective where $f_{i}$ is the projection of $X_{U_{i}}$ to $U_{i}$. Then $f_{i *}\left(\tilde{E}_{i}(m) \otimes \mathcal{O}_{X_{U_{i}}}\right)$ is a locally free $\mathcal{O}_{U_{i}}$-module of rank $H_{i}(m)$. Hence we have a morphism $\gamma_{i}: U_{i} \rightarrow G_{i}$, where $G_{i}$ is the Grassmann scheme $\operatorname{Grass}\left(V_{m} \otimes \mathcal{O}_{S}, H_{i}(m)\right)$. Set

$$
\Gamma=\Gamma^{0} \times{ }_{\left(Q^{0} \times \prod_{i=1}^{l} Q_{i}\right)}\left(Q^{0} \times \prod_{i=1}^{l} U_{i}\right) .
$$

Then, we obtain a homomorphism $\Psi: \Gamma-Z \times \prod_{i=1}^{l} G_{i}$.

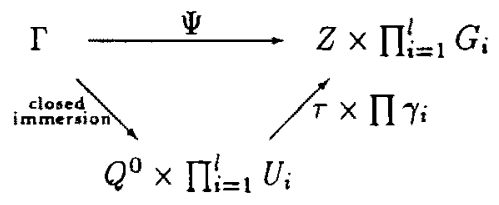

By virtue of Proposition 2.8, there exists an open subscheme $\Gamma^{s s}$ (or, $\Gamma^{s}$ ) of $\Gamma$ such that a geometric point $x$ of $\Gamma$ is contained in $\Gamma^{s s}$ (or, $\Gamma^{s}$ ) if and only if the corresponding parabolic sheaf $\left(\tilde{E}(m) \otimes \mathcal{O}_{X_{\Gamma}}, \bar{F}_{*}, \alpha_{*}\right) \otimes k(x)$ is strictly parabolic $e$-semi-stable (or, parabolic $e$-stable, respectively) and the homomorphism

$$
H^{0}(\phi \otimes k(x)): V_{m} \otimes k(x) \longrightarrow H^{\circ}(\tilde{E}(m) \otimes k(x))
$$

is an isomorphism.

Proposition 3.1 The morphism $\Psi: \Gamma^{s s}-Z \times \prod_{i=1}^{l} G_{i}$ is an immersion.

Proof. Let $V_{m} \otimes \mathcal{O}_{G_{i}} \rightarrow \mathcal{H}_{i}$ be the universal quotient on $G_{i}$ and let $J_{i}$ be the kernel of the quotient.

$$
0 \longrightarrow J_{i} \longrightarrow V_{m} \otimes \mathcal{O}_{G_{i}} \longrightarrow \mathcal{H}_{i} \longrightarrow 0
$$

Moreover, the universal quotient sheaf on $X_{Q}$ defines the following exact sequence.

$$
0 \longrightarrow \widetilde{K} \longrightarrow V_{m} \otimes \mathcal{O}_{X_{Q}} \longrightarrow \tilde{E}(m) \longrightarrow 0 .
$$

From these sequences, we have the following exact commutative diagram of $\mathcal{O}_{X_{Q \times G_{i}}}$-modules. (Set $\tilde{X}=X_{Q \times G_{i}}$ ) 


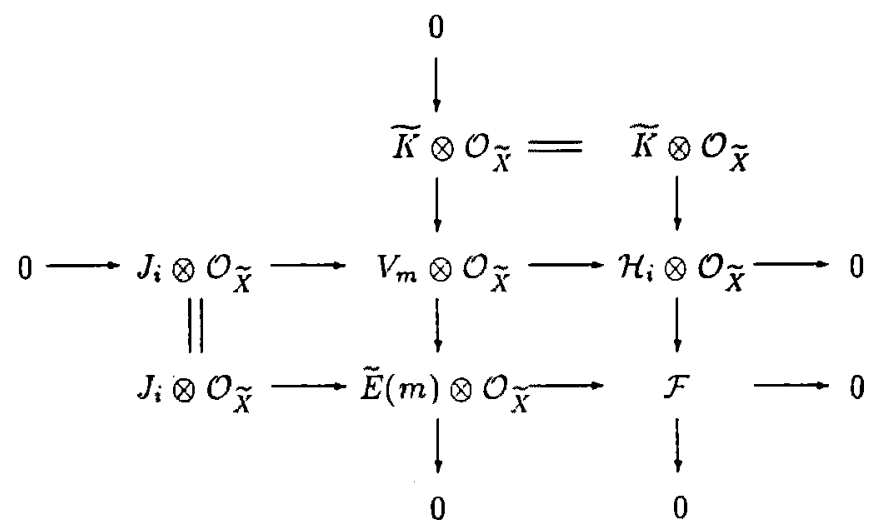

where $\mathcal{F}=V_{m} \otimes \mathcal{O}_{\tilde{X}} /\left(\widetilde{K} \otimes \mathcal{O}_{\tilde{X}}+J_{i} \otimes \mathcal{O}_{\tilde{X}}\right)$ that is not flat over $Q \times G_{i}$. Let us consider the Quot-scheme $Q \operatorname{uot}\left(\mathcal{H}_{i} \otimes \mathcal{O}_{\tilde{X}}, H_{i}[m]\right)$. Set $\bar{X}=X \times \operatorname{Quot}\left(\mathcal{H}_{i} \otimes\right.$ $\left.\mathcal{O}_{\tilde{X}}, H_{i}[m]\right)$. On $\bar{X}$, we have the universal quotient:

$$
\mathcal{H}_{i} \otimes \mathcal{O}_{\tilde{X}}-\tilde{\mathcal{F}} \longrightarrow 0
$$

Let $\delta$ be the following homomorphism of $\widetilde{K}_{\bar{K}} \otimes \mathcal{O}_{\bar{X}}$ to $\tilde{\mathcal{F}}$.

$$
\widetilde{K} \otimes \mathcal{O}_{X} \longrightarrow V_{m} \otimes \mathcal{O}_{\bar{X}} \longrightarrow \mathcal{H}_{i} \otimes \mathcal{O}_{X} \longrightarrow \tilde{\mathcal{F}}
$$

Then by Lemma 4.3 in [23], we have a closed subscheme $\Delta_{i}$ of $\operatorname{Quot}\left(\mathcal{H}_{i} \otimes\right.$ $\left.\mathcal{O}_{\tilde{X}}, H_{i}[m]\right)$ such that for every morphism $g: T \rightarrow \operatorname{Quot}\left(\mathcal{H}_{i} \otimes \mathcal{O}_{\tilde{X}}, H_{i}[m]\right)$, $\left(1_{X} \times g\right)^{*}(\delta)=0$ if and only if $g$ factors through $\Delta_{i}$. Then, on $X_{\Delta_{i}}$, the surjection (3.1.1) is factored as follows:

$$
\mathcal{H}_{i} \otimes \mathcal{O}_{X_{\Delta_{i}}} \longrightarrow \mathcal{F} \otimes \mathcal{O}_{X_{\Delta_{i}}} \stackrel{\lambda}{\longrightarrow} \tilde{\mathcal{F}} \otimes \mathcal{O}_{X_{\Delta_{i}}}
$$

Set

$$
\Delta_{i}^{0}=\left\{x \in \Delta_{i}|\quad \lambda|_{X_{x}} \text { is isomorphism }\right\} .
$$

Since $\Delta_{i}^{0}=\Delta_{i}-\pi_{\Delta_{i}}(\operatorname{Supp}(\operatorname{ker} \lambda)), \Delta_{i}^{0}$ is an open subset of $\Delta_{i}$. Let $\eta$ be the projection of $Q u o t\left(\mathcal{H}_{i} \otimes \mathcal{O}_{\tilde{X}}, H_{i}[m]\right)$ to $Q \times G_{i}$ and let $\widetilde{\Delta}_{i}$ be the scheme theoretic image of $\Delta_{i}$ by $\eta$. Since $\eta$ is proper, $\left.\eta\right|_{\Delta_{i}}: \Delta_{i} \rightarrow \tilde{\Delta}_{i}$ is surjective. Set

$$
\tilde{\Delta}_{i}^{0}=\left\{x \in \widetilde{\Delta}_{i} \mid \text { The Hilbert polynomial of }\left.\mathcal{F}\right|_{X_{x}} \text { is } H_{i}[m]\right\} \text {. }
$$

Then $\tilde{\Delta}_{i}^{0}$ is an open subset of $\tilde{\Delta}_{i}$. In fact, for all points $x$ of $\Delta_{i}-\Delta_{i}^{0}$, the Hilbert polynomial of $\left.\mathcal{F}\right|_{X_{x}}$ is not equal to that of $\left.\tilde{\mathcal{F}}\right|_{X_{x}}$. Hence,

$$
\begin{aligned}
\tilde{\Delta}_{i}^{0} & =\tilde{\Delta}_{i}-\eta\left(\Delta_{i}-\Delta_{i}^{0}\right) \\
& =\eta\left(\Delta_{i}^{0}\right) .
\end{aligned}
$$


Let $\iota: \amalg_{j} \tilde{\Delta}_{i j}^{0} \rightarrow \tilde{\Delta}_{i}^{0}$ be the flattenning stratification of $\mathcal{F} \otimes \mathcal{O}_{X_{\Delta_{i}^{0}}}$. (For the definition, see for example Lecture 8 in [15].) Then by the universality of $\amalg_{j} \tilde{\Delta}_{i j}^{0}$, $\eta: \Delta_{i}^{0} \rightarrow \tilde{\Delta}_{i}^{0}$ factors through $\amalg_{j} \widetilde{\Delta}_{i j}^{0} \rightarrow \widetilde{\Delta}_{i}^{0}$. Moreover, by the universality of $\Delta_{i}^{0}$, the morphism $\iota: \amalg_{j} \tilde{\Delta}_{i j}^{0} \rightarrow \tilde{\Delta}_{i}^{0}$ factors through $\eta$. Therefore there exists an isomorphism of $\Delta_{i}^{0}$ to $\amalg_{j} \tilde{\Delta}_{i j}^{0}$ and we obtain the following commutative diagram:

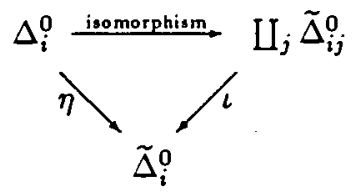

In particular, $\iota$ is proper. Therefore, all $\widetilde{\Delta}_{i j}^{0}$ are closed subscheme of $\widetilde{\Delta}_{i}^{0}$ and those images are open. Note that $\iota$ may not be an isomorphism, since, in general, $\tilde{\Delta}_{i}^{0}$ is not reduced. But we have known that $\eta: \Delta_{i}^{0} \rightarrow \tilde{\Delta}_{i}^{0}$ is a closed immersion.

By Lemma 4.3 in [23], we know that $\operatorname{Quot}\left(\mathcal{H}_{i} \otimes \mathcal{O}_{\tilde{X}}, H_{i}[m]\right)$ is a closed subscheme of $Q \times Q_{i}$. Hence, $\Delta_{i}^{0}$ is a subscheme of $Q \times Q_{i}$ and we obtain an immersion:

$$
\Delta_{1}^{0} \times_{Q} \Delta_{2}^{0} \times_{Q} \cdots \times_{Q} \Delta_{l}^{0} \longrightarrow Q \times \prod_{i=1}^{l} Q_{i} .
$$

Moreover, we have an immersion determined by $\eta: \Delta_{i}^{0} \rightarrow \tilde{\Delta}_{i}^{0}:$

$$
\Delta_{1}^{0} \times_{Q} \Delta_{2}^{0} \times_{Q} \cdots \times_{Q} \Delta_{i}^{0} \longrightarrow Q \times \prod_{i=1}^{l} G_{i} .
$$

We know that $\Gamma^{s s}$ is a subscheme of $Q \times \prod_{i=1}^{l} Q_{i}$ and the projection of $\Gamma^{s s}$ to $Q \times Q_{i}$ factored by $\Delta_{i}^{0}$. Hence, $\Gamma^{s s}$ is a subscheme of $\Delta_{1}^{0} \times Q \Delta_{2}^{0} \times Q \cdots \times{ }_{Q} \Delta_{l}^{0}$. Therefore, $\Gamma^{s s}$ is a subscheme of $Q \times \prod_{i=1}^{l} G_{i}$. Since $\nu: Q^{0} \rightarrow Z$ is an immersion and $\Gamma^{s s}$ is contained in $Q^{0} \times \prod_{i=1}^{l} Q_{i}, \Psi: \Gamma^{s s} \rightarrow Z \times \prod_{i=1}^{l} G_{i}$ is an immersion.

From now on, we assume that all $\alpha_{i}$ are rational numbers. Set $G=\operatorname{SL}\left(V_{m}\right)$ which acts on $\Gamma, Q, Q_{i}, Z$ and $G_{i} . \Psi$ is the $G$-morphism and $\Gamma^{s s}$ (or, $\Gamma^{s}$, respectively) is the $G$-invariant open set of $\Gamma$.

We shall define a $G$-linearized invertible sheaf on $Z \times \prod_{i=1}^{l} G_{i}$. Let $E_{\text {. }}$ be a member of $\mathcal{F}^{e}\left(H, H_{*}, \alpha_{*}\right)$ and let $\varepsilon_{i}=\alpha_{i+1}-\alpha_{i}$ for $i=1, \ldots, l$, where we put $\alpha_{l+1}=1$. Let $\mathcal{O}_{Z}(1)$ be a tautological invertible sheaf which has the canonical $G$-linearization and let $\mathcal{O}_{G_{i}}(1)$ be a tautological invertible sheaf on $G_{i}$ which has also the canonical $G$-linearization. Now, let $L$ be the $G$-linearized invertible sheaf:

$$
\mathcal{O}_{Z}\left(\operatorname{par}-P_{E_{\bullet}}(m)\right) \otimes \bigotimes_{i=1}^{l} \mathcal{O}_{G_{i}}\left(\varepsilon_{i}\right)
$$


The open set consisting of all semi-stable (or, stable ) points with respect to this $G$-linearization is denoted by $\left(Z \times \prod_{i=1}^{l} G_{i}\right)^{s s}$ (or, $\left(Z \times \prod_{i=1}^{l} G_{i}\right)^{s}$, respectively). Those are $G$-invariant open sets of $Z \times \prod_{i=1}^{l} G_{i}$.

Recall some facts on stable points of $Z$ or $G_{i}$. Let $x$ be a $K$-valued geometric point of $Z \times \prod_{i=1}^{l} G_{i}$. We denote the point of $Z(K)$ (or, $G_{i}(K)$ ) determined by $x$ by $T_{x}$ (or, $g_{i, x}$, respectively). We use the same symbol $g_{i, x}$ for the surjection $g_{i, x}: V_{m} \otimes K \rightarrow J_{i, x}$ which corresponds to $x$ and moreover, we denote its kernel by $W_{i, x} . T_{x}$ is identified with the homomorphism $T_{x}: \wedge^{r} V_{m} \otimes K \rightarrow H^{0}\left(L_{x}\right)$ where $L_{x}$ is the invertible sheaf corresponding to $\rho(x) \in P$. Each subspace $W$ of $V_{m} \otimes K$ has the maximal (or, minimal) $T_{x}$-dimension which is denoted by $\overline{\operatorname{dim}}_{T_{x}} W$ (or, $\operatorname{dim}_{T_{x}} W$, respectively). (See for the definitions of these dimensions, Definition $3.1 \mathrm{in} \mathrm{[23].)} \mathrm{In} \mathrm{general,} \underline{\operatorname{dim}}_{T_{x}} W \leq \overline{\operatorname{dim}}_{T_{x}} W$ and if equality holds, then it is denoted by $\operatorname{dim}_{T_{x}} W$.

Lemma 3.2 Let $x$ be a $K$-valued geometric point of $Z \times \prod_{i=1}^{l} G_{i}$. Assume that the point $T_{x}$ in $Z(K)$ has the following property:

$$
\text { For all subspaces } W \text { of } V_{m} \oslash K, \operatorname{dim}_{T_{x}} W=\overline{\operatorname{dim}}_{T_{x}} W \text {. }
$$

Then the point $x$ is semi-stable (or, stable) with respect to the $G$-linearized invertible sheaf $L$ if and only if for all non-trivial vector subspaces $W$ of $V_{m} \otimes K$, the following inequality holds:

$$
\begin{aligned}
& \operatorname{par}-P_{E_{*}}(m)\left(\operatorname{dim}_{T_{x}} W \cdot \operatorname{dim}\left(V_{m} \otimes K\right)-r \operatorname{dim} W\right) \\
& \quad+\sum_{i=1}^{l} \varepsilon_{i}\left(\operatorname{dim} W \cdot \operatorname{dim} W_{i, x}-\operatorname{dim}\left(V_{m} \otimes K\right) \cdot \operatorname{dim}\left(W_{i, x} \cap W\right)\right) \geq 0
\end{aligned}
$$

(or, $>0$, respectively).

Let $\sigma(W, x)$ be the left-hand side of the above inequality. Since $\operatorname{dim}\left(V_{m} \otimes\right.$ $K)=H(m)$ and $\operatorname{dim} W_{i, x}=H(m)-H_{i}(m)$. Hence, we have the following description of $\sigma(W, x)$ :

$$
\begin{aligned}
\sigma(W, x)= & \operatorname{dim} W\left(\sum_{i=1}^{l} \varepsilon_{i}\left(H(m)-H_{i}(m)\right)-\operatorname{par}-\chi\left(E_{-}(m)\right)\right) \\
& +H(m)\left(\operatorname{par}-P_{E_{*}}(m) \cdot \operatorname{dim}_{T_{x}} W-\sum_{i=1}^{l} \varepsilon_{i} \operatorname{dim}\left(W_{i, x} \cap W\right)\right) \\
= & \operatorname{dim} W\left(\left(1-\alpha_{1}\right) H(m)-\sum_{i=1}^{l} \varepsilon_{i} H_{i}(m)-H(m)+\sum_{i=1}^{l} \varepsilon_{i} H_{i}(m)\right) \\
& +H(m)\left(\operatorname{par}-P_{E_{*}}(m) \cdot \operatorname{dim}_{T_{x}} W-\sum_{i=1}^{l} \varepsilon_{i} \operatorname{dim}\left(W_{i, x} \cap W\right)\right) \\
= & H(m)\left(\operatorname{par}-P_{E_{*}}(m) \cdot \operatorname{dim}_{T_{x}} W-\sum_{i=1}^{l} \varepsilon_{i} \operatorname{dim}\left(W_{i, x} \cap W\right)-\alpha_{1} \operatorname{dim} W\right) .
\end{aligned}
$$


Proposition 3.3 1) $\Psi\left(\Gamma^{s s}\right) \subseteq\left(Z \times \prod_{i=1}^{l} G_{i}\right)^{s s}$.

2) $\Psi\left(\Gamma^{s}\right) \subseteq\left(Z \times \prod_{i=1}^{l} G_{i}\right)^{s}$.

3) If a point $x$ is in $\Gamma^{s s}$ but not in $\Gamma^{s}$, then $\Psi(x)$ is not in $\left(Z \times \prod_{i=1}^{l} G_{i}\right)^{s}$.

Proof. Let $x$ be a $K$-valued geometric point of $\Gamma^{s s}$ and $W$ be a non-trivial vector subspace of $V_{m} \otimes K$. Let the surjection $\phi_{x}: V_{m} \otimes \mathcal{O}_{X_{x}} \rightarrow E(m)$ and $E_{*}(m)$ correspond to $x \in \Gamma^{s s}$. By the definition of $\Gamma^{s s}, H^{0}\left(\phi_{x}\right): V_{m} \otimes K \rightarrow H^{0}(E(m))$ is an isomorphism. Set $F(m)=\phi_{x}\left(W \otimes \mathcal{O}_{X_{x}}\right)$. Then we have

$$
\operatorname{dim}_{T_{\psi(x)}} W=\operatorname{rk}(F) .
$$

Since $W$ and $H^{0}(F(m))$ are subspaces of $V_{m} \otimes K \cong H^{0}(E(m)), W \subset H^{0}(F(m))$, hence, we have

$$
\operatorname{dim} W \leq h^{0}(F(m)) .
$$

The subspace $W_{i, \Psi(x)}$ of $V_{m} \otimes K^{\prime}$ is identified with $H^{0}\left(F_{i+1}(E)(m)\right)$ by $H^{0}\left(\phi_{x}\right)$. Since

$$
H^{0}\left(\left(F_{i+1}(E) \cap F\right)(m)\right)=H^{0}\left(F_{i+1}(E)(m)\right) \cap H^{0}(F(m)),
$$

we have

$$
\operatorname{dim} W_{i, \Psi(x)} \cap W \leq h^{0}\left(\left(F_{i+1}(E) \cap F\right)(m)\right) .
$$

Therefore, we obtain

$$
\begin{aligned}
\sigma(W, \Psi(x)) \geq & H(m)\left(\text { par- } P_{E_{0}}(m) \cdot \operatorname{rk}(F)\right. \\
& \left.-\left(\alpha_{\mathbf{1}} h^{0}(F(m))+\sum_{i=1}^{1} \varepsilon_{i} h^{0}\left(\left(F_{i+1}(E) \cap F\right)(m)\right)\right)\right) \\
= & H(m)\left(\operatorname{par}-P_{E_{0}}(m) \cdot \operatorname{rk}(F)-\int_{0}^{1} h^{0}\left(F_{\alpha}(m)\right) d \alpha\right) .
\end{aligned}
$$

By virtue of Proposition 2.5 and Lemma 3.2, the assertions 1) and 2) have been proved. Let $E_{*}^{\prime}$ be a parabolic subsheaf of $E_{*}$ which satisfies the last condition in Proposition 2.5. Set $W=H^{0}\left(E^{\prime}(m)\right)$. Then we have

$$
H^{0}\left(\left(E^{\prime} \cap F_{i+1}(E)\right)(m)\right)=W \cap W_{i, \Psi(x)} .
$$

Since $E^{\prime}(m)$ is generated by its global sections, $\operatorname{dim}_{T_{\Psi_{(x)}}} W=\operatorname{rk}\left(E^{\prime}\right)$. Hence,

$$
\sigma(W, \Psi(x))=H(m)\left(\operatorname{par}-P_{E_{0}}(m) \cdot \operatorname{rk}\left(E^{\prime}\right)-\int_{0}^{1} h^{0}\left(E_{\alpha}^{\prime}(m) d \alpha\right)=0 .\right.
$$

So, $\Psi(x)$ is not in $\left(Z \times \prod_{i=1}^{l} G_{i}\right)^{s}$.

Let $\tilde{\Gamma}^{s s}$ be the scheme theoretic image of $\Gamma^{s s}$ in $\left(Z \times \prod_{i=1}^{l} G_{i}\right)^{s s}$. Then by virtue of Theorem 4 of [18], there exists a good quotient $\xi: \tilde{\Gamma}^{s s} \rightarrow Y$ and $Y$ is projective over $S$. Set $\bar{M}_{e}=Y-\xi\left(\tilde{\Gamma}^{s s}-\Gamma^{s s}\right)$. Then $\bar{M}_{e}$ is quasi-projective over $S$. Moreover $\bar{M}_{e}$ contains $M_{\mathrm{e}}=\xi\left(\Gamma^{s}\right)$ as an open subscheme. By the similar arguments as in $\$ 5$ in $[8]$, we have 
Theorem 3.4 $M_{e}$ is a coarse moduli scheme of par- $\Sigma_{D / X / S}^{H_{*}, \alpha, e}$, that is, $M_{e}$ has the following properties.

1) For each geometric point $s$ of $S$, there exist a natural bijection:

$$
\theta_{s}: \operatorname{par}-\Sigma_{D / X / S}^{H_{*}, \alpha_{*}, e}(k(s)) \longrightarrow M_{e}(k(s))
$$

2) For $T \in(S c h / S)$ and a flat family of parabolic sheaves $E_{ \pm}$on $X_{T} / T$ such that $E_{*}$ has the property (1.12.1) and for every geometric point $t$ of $T,\left.E_{*}\right|_{X_{*}}$ is parabolic e-stable, then there exists a morphism

$$
f_{E}^{e}: T \longrightarrow M_{e}
$$

such that for all points $t$ in $T(k(s)), f_{E_{*}}^{e}(t)=\theta_{s}\left(\left.E_{*}\right|_{X_{\mathrm{t}}}\right)$ Moreover, for a morphism $g: T^{\prime} \rightarrow T$ in $(S c h / S)$,

$$
f_{E_{\bullet}}^{e} \circ g=f_{(1 \times \times g)^{*}}^{e}\left(E_{\bullet}\right)
$$

3) If $M^{\prime} \in(S c h / S)$ and maps

$$
\theta^{\prime},: \operatorname{par}-\Sigma_{D / X / S}^{H *, \alpha, e}(k(s)) \longrightarrow M^{\prime}(k(s))
$$

have the above property 2), then there exists a unique $S$-morphism $\Upsilon$ of $M_{e}$ to $M^{\prime}$ such that $\Upsilon(k(s)) \circ \theta_{s}=\theta^{\prime}{ }_{s}$ and $\Upsilon \circ f_{E}^{e}=f^{\prime}{ }_{E}$, for all geometric points $s$ of $S$ and for all $E_{*}$, where $f^{\prime} E_{*}$ is a morphism given by the property 2 ) for $M^{\prime}$ and $\theta^{\prime}{ }_{s}$.

By the similar arguments as in $\S 5$ of [8], we know that there exists a unique morphism $\nu_{e, e^{\prime}}$ of $M_{e}$ to $M_{e^{\prime}}$ if $e \leq e^{\prime}$. Moreover, $M_{e}$ can be regarded as an open subscheme of $M_{e^{\prime}}$ through $\nu_{e, e^{t}}$. Taking inductive limit of $\left\{M_{e}\right\}$, an $S$-scheme $M_{D / X / S}^{H_{*} \alpha_{*}}$ is obtained. By the same proof as Theorem 5.6 of [8], we have

Theorem 3.5 The functor par- $\Sigma_{D / X / S}^{H_{*}, \alpha_{*}}$ has a coarse moduli scheme $M_{D / X / S}^{H_{*}, \alpha_{*}}$ in $(S c h / S)$. Moreover, $M_{D / X / S}^{H_{*}, \alpha_{*}}$ is separated and locally of finite type over $S$.

For the functor par- $\bar{\Sigma}_{D / X / S}^{H_{*}, \alpha_{*}, e}$, by the similar argument as in $\S 4$ of [9], we have Theorem $3.6 \bar{M}_{e}$ has the following properties.

1) For each geometric point $s$ of $S$, there exists a natural surjection :

$$
\bar{\theta}_{s}: \operatorname{par}-\bar{\Sigma}_{D / X / S}^{H_{*}, \alpha_{*}, e}(k(s)) \longrightarrow \bar{M}_{e}(k(s)) .
$$


2) For $T \in(S c h / S)$ and a flat family of parabolic sheaves $E_{\text {. on }} X_{T} / T$ such that $E_{*}$ has the property (1.12.1) and for every geometric point $t$ of $T,\left.E_{*}\right|_{X_{\mathrm{t}}}$ is strictly parabolic e-semi-stable, then there exists a morphism

$$
f_{E .}^{e}: T \longrightarrow \vec{M}_{e}
$$

such that for all points $t$ in $T(k(s)), f_{E_{*}}^{e}(t)=\bar{\theta}\left(\left[\left.E_{*}\right|_{X_{t}}\right]\right)$ where [.] means the equivarence class defined by (1.12.2). Moreover, for a morphism $g: T^{\prime} \rightarrow T$ in $(S c h / S)$, we have

$$
f_{E}^{e} \circ g=f_{\left(1_{X} \times g\right)^{*}}^{e}\left(E_{\bullet}\right)^{*}
$$

\section{References}

[1] A. Altman and S.Kleiman, Compactifying the Picard Scheme, Adv. in Math. 35 (1980), 50-112.

[2] U. N. Bhosle, Parabolic vector bundles on curves, Ark. Mat. 27 (1989), $15-22$.

[3] D. Gieseker, On the moduli of vector bundles on an algebraic surface, Ann. of Math. 106 (1977), 45-60.

[4] A. Grothendieck and J.Dieudonné, Élements de Géométrie Algébrique, Ch. I, II, III, IV, Publ. Math. I.H.E.S. Nos. 4, 8, 11, 17, 20, 24, 28 and 32.

[5] A. Grothen dieck, Techniquis de construction et théorèmes d'existence en géométrie algébrique, IV: Les schémas de Hilbert, Sem. Bourbaki, t.13, $1960 / 61, n^{\circ} 221$.

[6] N. J. Hitchin, The self-duality equations on a Riemann surface, Proc. London Math. Soc. (3) 55 (1987), 59-126.

[7] N. J. Hitchin, Stable bundles and integrable systems, Duke Math. J. 54 (1987), 01-114.

[8] M. Maruyama, Moduli of stable sheaves, I, J. Math. Kyoto Univ. 17 (1977), 91-126.

[9] M. Maruyama, Moduli of stable sheaves, II, J. Math. Kyoto Univ. 18 (1978), 557-614.

[10] M. Maruyama, On boundedness of families of torsion free sheaves, J. Math. Kyoto Univ. 21 (1981), 673-701.

[11] M. Maruyama, Openness of a family of torsion free sheaves, J. Math. Kyoto Univ. 16 (1976), 627-637. 
[12] V. B. Mehta and A. Ramanathan, Semistable sheaves on projective varieties and their restriction to curves, Math. Ann. 258 (1982), 213-224.

[13] V. B. Mehta and C. S. Seshadri, Moduli of vector bundles on curves with parabolic structures, Math. Ann. 248 (1980), 205-239.

[14] D. Mumford, Geometric Invariant Theory, Springer-Verlag, BerlinHeidelberg-New York, 1965.

[15] D. Mumford, Lectures on Curves on an Algebraic Surface, Annals of Math. Studies 59, Princeton U. Press, Princeton, 1966.

[16] M. S. Narasimhan and C. S. Seshadri, Stable and unitary vector bundles on a compact Riemann surface, Ann. of Math. 82 (1965), 540-567.

[17] N. Nitsure, Moduli spaces for stable pairs on a curve, Preprint (1988).

[18] C. S. Seshadri, Geometric reductivity over arbitrary base, Adv. in Math. 26 (1977), 225-274.

[19] C. S. Seshadri, Moduli of vector bundles on curves with parabolic structures, Bull. Amer. Math. Soc. 83 (1977), 124-126.

[20] C. S. Seshadri, fibrés vectoriels sur les courbes algébriques, astérisque 96 (1982).

[21] S. Shatz, The decomposition and specialization of algebraic families of vector bundles, Compositio Math. 35 (1977), 163-187.

[22] C. T. Simpson, Moduli of representations of the fundamental group of a smooth projective variety, Preprint, Princeton Univercity.

[23] K. Yokogawa, Moduli of stable pairs, to appear in J. Math. Kyoto Univ. 